\title{
Cinchona amazonica Standl. (Rubiaceae) no estado do Acre, Brasil ${ }^{1}$
}

Cinchona amazonica Standl. (Rubiaceae) in the state of Acre, Brazil

Resumo: $\bigcirc$ presente estudo trata da dendrologia, distribuição geográfica e status de conservação de Cinchona amazonica Standl., plantas de interesse medicinal que ocorrem no estado do Acre, Brasil. A pesquisa consistiu em trabalhos de campo para a coleta de material botânico, levantamento e estudo das exsicatas disponíveis nos herbários nacionais e internacionais da América do Sul, revisão bibliográfica das espécies do gênero na literatura e nos sites especializados. Os resultados apresentam a descrição da espécie com a sua ilustração e mapeamento dos especímenes, bem como determinação da sua situação populacional.

Palavras-chave: Cinchona. Dendrologia. Ditribuição geográfica. Status de conservação. Acre.

Abstract: The present research is about dendrology, geographic distribution and status of conservation of Cinchona amazonica Standl. with are plants with medical interest; they grow in the state of Acre, Brazil. The research consisted in: field work for the collection of botanical material, in the survey and study of the available exsiccates in the national and international herbariums of the South America. It has been done a bibliographical review of the species of this genera in literature and the specialized sites The results present the description of the species with their illustration and mapping, as well as determination of their population situation.

Keywords: Cinchona. Dendrology. Geographic distribution. Conservation status. Acre

\footnotetext{
I Universidade Nacional Agraria La Molina. Faculdad de Ciencias Florestales. Lima, Peru (pazpolli@lamolina.edu.pe).

II Universidade de São Paulo. Escola Superior de Agricultura Luiz de Queiroz. Piracicaba, São Paulo, Brasil (mtomazel@asalq.usp.br).

1 Financiado com bolsa de estudo da Organização de Estados Americanos (OEA).
}

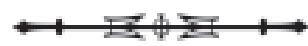




\section{INTRODUÇÃO}

O estado do Acre, na Amazônia Ocidental, possui 93\% do seu território coberto de florestas pluviais e constitui o maior patrimônio genético vegetal da região norte do Brasil (IMAC, 1991). A redução da cobertura vegetal ( $5 \%$ ao ano), conseqüência da exploração madeireira e ampliação da fronteira agropecuária, pode levar à extinção de parte da flora ainda desconhecida e considerada como fonte potencial de produtos medicinais, alimentares e florestais (MENEZES, 1994).

Apesar da existência de uma florescente indústria farmacêutica que pesquisa e desenvolve continuamente novos medicamentos, a Organização Mundial da Saúde (OMS) estima que 80\% da população mundial depende da medicina tradicional e $85 \%$ desta utiliza as plantas medicinais, seus extratos vegetais e princípios ativos (IUCN, 1993).

Das inúmeras plantas com substâncias químicas utilizadas nos tratamentos terapêuticos, estão incluídas as espécies do gênero Cinchona, fonte de quinina para as febres decorrentes ou malária, e quinidina para doenças cardíacas (WARHURST, 1987; ZEVALLOS, 1989; OCAMPO-SANCHEZ, 2000).

Os estudos sobre as espécies de Cinchona e de outras plantas nos países andinos da Amazônia, têm sido relativamente intensificados pela importância farmacêutica e econômica dessas espécies (OCAMPOSÁNCHEZ, 2000) sendo, porém, reduzidos no Brasil (SCHULTES, 1979; DELPRETE; CORTEZ, 2002).

Com base no exposto, o presente trabalho tem como objetivos a caracterização dendrológica de Cinchona amazonica Standl., única espécie do gênero Cinchona encontrada no estado do Acre, Brasil; a definição do seu padrão de distribuição geográfica; e a determinação do seu status de conservação ou situação populacional, para conhecer o grau de retração ou expansão das plantas da espécie na região, contribuindo para aumentar o conhecimento da flora amazônica.

\section{REVISÃO DE LITERATURA}

\section{Aspectos taxonômicos}

O gênero Cinchona tem sido intensamente estudado desde a sua descoberta para a cura da malária, no final do século XVI. No mundo, foram descritas cerca de 38 a 50 espécies de árvores e 150 variedades de arbustos perenes do gênero, nas escarpas mornas e úmidas dos Andes, em altitudes de 1.500 a 2.500 m, assim como na Amazônia (STEYERMARK, 1974; STELL, 1982; GENTRY, 1993; BREMER; ANDREASSEN; OLOSSON, 1995). São encontrados 167 registros de nomes científicos para o gênero (REA, 1995), dos quais 23 foram reconhecidos por Anderson (1998).

De acordo com Cronquist (1988), o gênero Cinchona pertence à família Rubiaceae, ordem Rubiales, Subclasse Asteridae, classe Magnoliopsida, Divisão Magnoliophyta. A família foi segregada da Ordem Gentianales em função da classificação filogenética de A. Engler (HUTCHINSON, 1967) e incluída por Robbrecht (1988) na subfamília Cinchonoideae. No entanto, desde DeCandole (1797) até Anderson (1995), situa-se na Tribu Cinchoneae, que reúne os gêneros com sementes aladas. Bremer, K., Bremer, B. e Thulin (1998), de acordo com a análise cladística realizada, reclassificou a família Rubiaceae na ordem Gentianales sob uma sustentação monofilética, voltando à ordem original, pertencente ao grupo mais elevado Euasteridas I.

\section{Aspectos ecológicos e distribuição geográfica}

Espécies do gênero Cinchona ocorrem em climas quentes e úmidos, com precipitação abundante e persistente, com nebulosidade o ano todo, nas zonas altas com topografia íngreme e nas partes baixas das colinas, quase sem influência do clima. Encontram-se em áreas com temperaturas de 6,5 a $24,9^{\circ} \mathrm{C}$ e precipitações de 790 a 3.900 mm, alcançando índices máximos no verão (ZEVALLOS, 1989; OCAMPO-SÁNCHEZ, 2000).

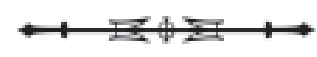


Em geral, ocorrem em regiões com solos de origem residual ou aluvial, profundos, de reação ácida e bem drenados, com textura areno-argilosa, finos e com abundante matéria orgânica (BARQUERO, 1986; ZEVALLOS, 1989; OCAMPO-SÁNCHEZ, 2000).

De origem americana, segundo Anderson (1998), as espécies de Cinchona e sua ancestral têm provável ocorrência nas florestas de neblina dos Andes Centrais quase ao final do Cretáceo, quando completou-se a formação das montanhas de baixas elevações. É nativa das florestas úmidas, geralmente acima de $100 \mathrm{~m}$, podendo chegar até 3.000 m (BURGER, 1993; GUPTA, 1995; OCAMPO-SÁNCHEZ, 2000) entre as latitudes de $10^{\circ} \mathrm{Ne} 19^{\circ} \mathrm{S}$ (VAN-HARTEN, 1987). Na Amazônia brasileira somente C. amazonica tem sido reportada até o nível do mar (ZEVALLOS, 1989).

\section{Importância florestal, medicinal e econômica}

As espécies do gênero Cinchona são consideradas, universalmente, como as que salvaram a humanidade da malária ou paludismo. Em 1643 foi reportada pelos jesuítas, pela primeira vez na Europa, sendo utilizada marcadamente durante as duas Guerras Mundiais (ZEVALLOS, 1989; VERVEEN, 1984). Compreende várias espécies chamadas vulgarmente de 'cascarilla, árvore da quina ou árvore do quinino', que contém quinina (alcalóide com propriedades antifebrífugas) na sua casca, das quais foram extraídas grandes quantidades, no Peru e na Bolívia. Em1820, após o isolamento do alcalóide quinina, seguiu-se uma intensa competição para se identificar a espécie com o mais alto teor desse composto, o que causou a extinção de populações de plantas de cinchonas selvagens no início do século XIX e induziu o estabelecimento de plantações das espécies. Posteriormente, com o sucesso dos holandeses no cultivo de Cinchona calisaya, Java tornou-se o centro mundial de produção de quinina (CARVALHO; KRUG, 1944; ANDERSSON, 1998).

As espécies de Cinchona, de reconhecida importância desde o século XVII são, atualmente, cultivadas em inúmeras regiões tropicais, com produção anual de
5.000 a 10.000 t de casca e 400 a 500 t de alcalóide, com $60 \%$ da produção mundial destinada à indústria farmacêutica e 30 a 50\% à indústria agroalimentar (REA, 1995; VERPOORTE; SCHRIPSEMA, VANDER-LEER, 1988).

Os compostos químicos das espécies do gênero Cinchona são os alcalóides quinólicos (quinina, quinidina, dihidroquinidina, cinconidina, cinconina e dehidrocinconina) e indólicos (dihidroquinamina e 3 epi-dihidroquinamina), com a quinina apresentando a mais alta atividade antimalárica (VERPOORTE; SCHRIPSEMA; VAN-DER-LEER, 1988). A quinina e a quinidina são utilizadas, ao nível do miocárdio, contra problemas de eretismo cardíaco e anginas precordiais; o clohidrato de quinina para esclerosis de origem hemorroidal; o escorbato de quinina, associado à vitamina $B$, para o tabagismo e para cãibra, ademais é analgésica e antipirética (REA, 1995). A quinina é usada como aditivo amargo nos alimentos e bebidas (BRUNETON, 2001) e na indústria química nas catálises e imobilizações de matrizes poliméricas (VERPOORTE; SCHRIPSEMA, VAN-DER-LEER, 1988). A quinidina como antifibrilante é adequada ao tratamento preventivo da taquicardia, justificando que 30 a 50\% da produção de quinina é transformada por meios físicomecânicos em quinidina (PRINZ, 1990; BRUNETON, 2001). Os alcalóides quinolínicos apresentam atividade contra o vírus da batata (VERPOORTE; SCHRIPSEMA, VAN-DER-LEER, 1988) e os indólicos do tipo cincofilinas contra as bactérias gram-positivas (REA, 1995).

\section{MATERIAL E MÉTODOS}

Foram revisadas as coleções de Cinchona amazonica Standl. dos seguintes herbários no Brasil: ESA, HPZ, HRCB, IAN, INPA, MG, R, RB, SP, SPF, UEC e UNBA; na Bolívia: LPB; e no Peru: AMAZ, MOL e USM. Os especímenes examinados no Brasil, foram: Acre [(].M.A. de Souza 116-SP, 6/14/890) (09²0'00" 69०00'00" B. A. Krukoff 5520-RB, 8/15/33) (Mâncio Lima,

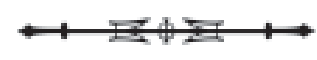




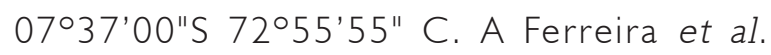
10962-UFAC, 3/25/92) (Mâncio Lima 07036'00" 72057'00" D.C. Doly 9047-UFAC, 5/13/96) (Cruzeiro do Sul, Steward W. $12801-M G, 5 / 8 / 71$ ) (Rio Branco, Fróes R. 22962-IAN, 2/6/48) (Cruzeiro do Sul, Froes R. 137-IAN, 2/12/76)], Amazonas (Humaitá 0745'00"S 53016'00" L.O.A. Teixeira et al. 1171-RB, 6/17/82) (Manaus, S.G. Egler et al. 1588-SPF, 00/03/1993) (Manaus, Leras et al. sn-MG, 11/21/73) (Froes R. 23286-IAN, 6/24/48) (Fróes R. 21682-IAN, 10/3/46) (Manaus, Leras E. 19585-INPA, 11/21/77) (Manaus, Silva M. 334-INPA, 7/10/72) (Manaus, Prance G. 20481-INPA, 3/13/74) (Manaus, sc, sn-R11/21/73)], Pará [dos Reis Cordeiro M. 2247MG, 10/31/91], Roraima [(G.T. Prance et al. 20001 12/3/73) (N. A. Rosa 311-RB, 1/26/75)], Colômbia [(Amazonas, Acero E. 939-INPA, 2/9/79) (Comisaría del Vaupés, 69045'00" Fernandez A. 2140-IAN, 2/4/

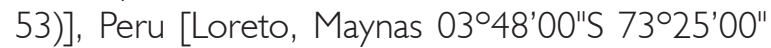
Vasquez R. 4577-AMAZ, 11/9/83) (Pasco, Oxapampa 10³5'00"S 75²8'00" Smitth D. 1635-AMAZ, 5/22/82) (Pasco, Oxapampa Daza A. 1855AD-MOL 27/11/2001)].

A amostragem de campo estendeu-se à maioria dos municípios do estado do Acre (Acrelândia, Assis Brasil, Brasiléia, Bujari, Capixaba, Cruzeiro do Sul, Epitaceolândia, Feijó, Mâncio Lima, Manoel Urbano, Plácido de Castro, Porto Walter, Rio Branco, Rodrigues Alves, Senador Guiomard, Sena Madureira, Tarauacá e Xapuri), considerando a acessibilidade, probabilidade de localização das espécies de interesse, fundamentada na revisão do material nos herbários, consulta bibliográfica e informações coletadas dos moradores locais. Nas coletas botânicas foram incluídas as informações sobre o grupo ecológico e dados sobre a regeneração natural (Figura 1).

A identificação das coleções botânicas foi realizada de acordo com a metodologia de Radford et al. (1974) e, para a caracterização dendrológica e ilustração, foram considerados os aspectos gerais (hábito, ramificação, tronco, base do tronco, raízes, diâmetro e altura), casca (cor, odor, sabor, ritidoma, exsudado); folha (tipo, forma, ápice, base, estípulas, glândulas), inflorescência (tipo, tamanho, diâmetro), flores (cálice, corola, androceu e gineceu), frutos (tipo, forma, cor, consistência, deiscência etc.) e sementes (tamanho, presença de testa, viabilidade).

Registros de outras coletas em base de dados, sítios na internet e bibliografia especializada referentes as espécies que ocorrem no Brasil e no exterior foram obtidas no [W3TROPICOS], Missouri Botanical Garden (MO), New York Botanical Garden (NYBG), International Plant Name Index Query e Flora Brasilensis.

A distribuição geográfica das espécies do gênero no Brasil e na América Latina foi obtida aplicandose o programa Arc View 3.1, tratados no sistema de informação geográfica (SIG). As coordenadas geográficas foram obtidas das revisões das exsicatas dos herbários, de fontes bibliográficas e de outras observações feitas durante o trabalho de campo. As categorizações foram baseadas nas propostas do Centro de Datos para a Conservação (CDC, 1991).

A determinação do status de conservação foi obtido aplicando-se a metodologia da IUCN $(1998,2002)$ adaptada para as condições da região, classificando a situação populacional das espécies de Uncaria em [1] extinto (EX), [2] extinto no estado silvestre (EW), [3] perigo crítico (CR), [4] em perigo (EN), [5] vulnerável (VU), [6] quase ameaçada (NT), [7] preocupação menor (LC), [8] dados insuficientes (DD), [9] não-avaliado (NE).

\section{RESULTADOS E DISCUSSÃO}

Cinchona amazonica Standl. Pub. Field Col. Mu. Bot. Ser. 8(5): 334. 1931. Tipo: Peru: Loreto: on the Amazon River in forest, 24 Jul 1929, Llewelyn Williams 1747 (HT: F 604562). Sin: Cinchonopsis amazonica (Standl.) L. Andersson, publi. Ann. Missouri Bot. Gard.: 82(3): 424. 1995. Nome vulgar: desconhecido.

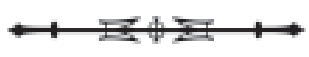




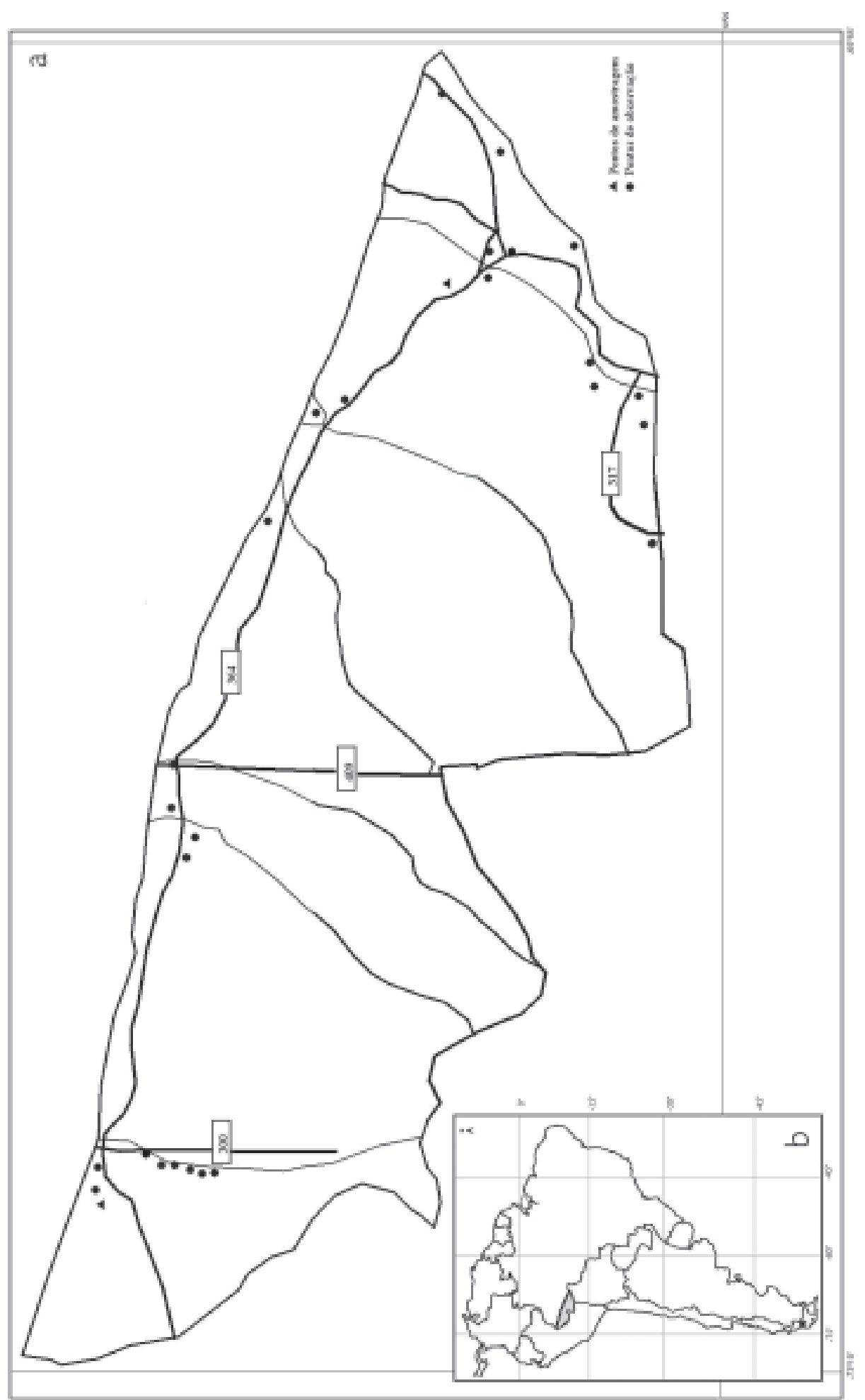

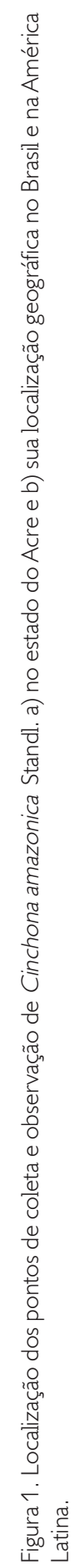

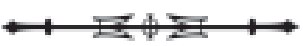




\section{Caracterização dendrológica}

Árvore de modo geral semi-tolerante à luz, alcançando mais de $15 \mathrm{~m}$ de altura, 18 a $40 \mathrm{~cm}$ de diâmetro; copa grande e bífida; fuste cilíndrico; casca externa marrom-bege, com manchas verde-escuras, aparentemente fissurada, ritidoma não-evidente; casca interna vermelho-claro a rosa-amarelado, de textura fibro-arenosa. Ramos terminais de seção cilíndrica ou semi-quadrada até $10 \mathrm{~mm}$ de diâmetro, verdeamarronzado a marrom-claro; folhas terminais lanceoladas, glabras ou pilosas quando jovens, pequena, hirsutas, bege a verde-bege. Caule quando seco, pode ser oco. Folhas simples, opostas semicruzadas, abovadas, elíptico-oblongas ou elípticas, 14 a $27 \mathrm{~cm}$ de comprimento, 5 a $12 \mathrm{~cm}$ de largura; borda ou margem inteira a ligeiramente sinuosa; ápice obtuso; base atenuada, agudo-redonda a aguda; nervura pinatinérvea oblíqua, com 14 a 15 pares de nervuras, cartáceas a coriáceas; face abaxial verdeescuro e adaxial verde-claro brilhante; tricomas na face abaxial, das axilas das nervuras, finos, retos e densos; pecíolo de 3 a $6,5 \mathrm{~cm}$ de comprimento, 5 mm de diâmetro; 1 par de estípulas interpeciolares, abovadas, coriáceas, caducas, deixando uma cicatriz conspícua nos ramos terminais; folhas jovens em geral maiores e com abundantes tricomas. Inflorescências em panículas terminais, grandes, até $50 \mathrm{~cm}$ de comprimento. Caule marrom a marrom-claro, com tricomas levemente densos e curtos. Flores bissexuais com até $3 \mathrm{~cm}$ de comprimento; brácteas até 5 a $6 \mathrm{~mm}$ de comprimento, tricomas curtos e hirsutos, densos na face adaxial e raros na abaxial; coléteres marrons quando secos na base da face adaxial, acompanhados com pêlos longos, finos e densos, ocupando uma terceira parte; hipanto com cálice adnato, $6 \mathrm{~mm}$ de comprimento; cálice persistente, gamossépalo, cilíndrico, 5 lobado; tubo de $0,5 \mathrm{~mm}$ de comprimento, bordo até $0,5 \mathrm{~mm}$ de comprimento, densamente pilosos em toda a superfície; corola hipocrateriforme ou infundibuliforme, 5 lobulada, lóbulos alongados, com pêlos muito curtos e finos em toda a superfície, reflexos, agudos; tubo até $9 \mathrm{~mm}$ de comprimento, esbranquiçado, com a base ligeiramente escura, bordo até $10 \mathrm{~mm}$ de comprimento, branco ou amarelo; androceu com 5 estames adnatos à base da corola, com filetes curtos de 3 a 3,5 mm de comprimento, com anteras bitecas, dorsifixas e de deiscência longitudinal; gineceu de ovário ínfero, 2-carpelar, 2-locular, placentação axilar, presença de disco contínuo e em forma de taça, estilo terminal engrossados de 8 a $10 \mathrm{~mm}$ de comprimento e estigma bífido de 1,5 mm cada. Fruto seco tipo cápsula, cilíndrica alongada, 2-valvar de 3 a $8 \mathrm{~cm}$ de comprimento e 3 a $6 \mathrm{~mm}$ de largura cada, geralmente com baixa densidade de pêlos mais ou menos curtos, deiscência longitudinal, abrindo-se da base até o ápice. Sementes aladas de 1 a $2 \mathrm{~cm}$ de comprimento e 3 a 4 mm de largura (Figura 2).

\section{Aspectos taxonômicos}

Cinchona amazonica apresentou complexidade quanto à sua identificação, devido às variações nos materiais botânicos coletados e analisados segundo as descrições da bibliografia especializada baseadas, principalmente, na consistência, pubescência e na cor das folhas, características das estruturas florais e reprodutivas e, também, devido a semelhança com outras espécies de gêneros relacionados à familia (Landerbergia, Remijia e Macrocnerum) (ANDERSSON; PERSSON, 1991; ANDERSSON, 1995; REA, 1995; ZEVALLOS, 1998; PINO, 2002). A revisão das Tribos e gêneros da Cinchoneae feita por Andersson (1995) permitiu a diferenciação pela grande variabilidade morfológica existente nas espécies de Rubiaceae e, ainda maior, em Cinchona. $\bigcirc$ autor estabeleceu sinonímias, excluiu e incorporou espécies e criou novos gêneros como o Cinchonopsis, com uma única espécie Cinchonopsis amazonica, a partir da observação de somente 2 exsicatas: Fernández e Sanoja 4964-NY e Fosbert 38838-NY, com Cinchona amazonica sendo o basinimio. A deiscência do fruto, a pubescência e a cor da corola foram as características de diferenciação mais marcantes utilizadas por Andersson (1995) que podem, no entanto, variar

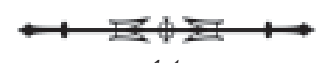




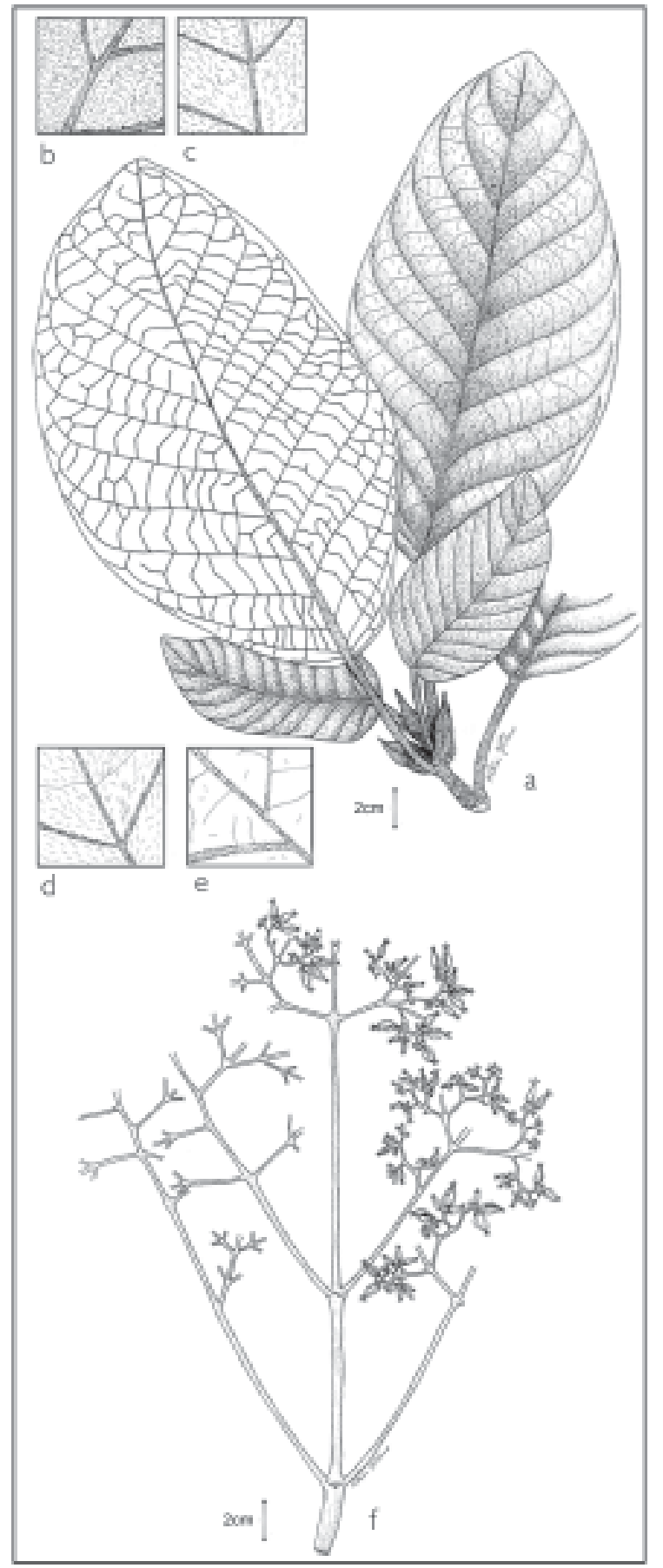

Figura 2. Cinchona amazonica Standl. a) galho terminal; b) e c) pubescência face abaxial e adaxial de folha jovem; d) e e) pubescência face abaxial e adaxial de folha velha; f) inflorescência (P. A. ZEVALLOS, 2925) em função da idade e são, geralmente, resultado das adaptações às condições ecológicas do local de ocorrência das plantas. No caso da deiscência dos frutos, quando estes são coletados verdes ou imaturos, verifica-se que os tecidos da sua parte apical não completaram o desenvolvimento e ao secarem fendem nessa região de forma acrópeta e que, nos frutos maduros, a fenda ocorre na sua base (BARCELÓ et al, 2001), demostrando a importância da coleta de órgãos reprodutivos maturos. Considera-se, também, que o número de exsicatas analisadas para a definição de Cinchonopsis não é representativo, pois não incorpora toda a variabilidade das características vegetativas e reprodutivas do taxon. Há necessidade, portanto, de uma revisão das exsicatas dos herbários da América Latina e, principalmente, de envidar esforços para a coleta de novos materiais botânicos na área de ocorrência da espécie na Amazônia. O lenho de Cinchona amazonica, coletado no estado do Acre (ZEVALLOS, 2004), mostrou características anatômicas semelhantes às descritas para Cinchona por Détienne e Jacquet (1993). A constatação de somente 24 exsicatas nos herbários indica uma escassa ou não representativa amostragem do gênero, justificando a necessidade de novas coletas botânicas nas regiões estudadas e a manutenção, no presente trabalho, da denominação Cinchona amazônica Standl.

Plantas de Cinchona delessertiana Standl. e Cinchona humboldtiana Roem. \& Schult foram encontradas na Amazônia Brasileira (MG-55212 \& MG-0149659) e Peruana (ZEVALLOS, 1989) e sua ocorrência é provável, sobretudo, na Cordilheira da Serra Divisor, acima de $600 \mathrm{~m}$ de altitude.

\section{Distribuição geográfica}

Esta espécie encontra-se distribuída nas vertentes da bacia Amazônica, podendo ser encontrada entre as faixas latitudinais (03010'00"N-13017'00"S), longitudinais (53016'00"W-75028"00"W) e altitudinais (70 a 1970 m). Na América do Sul, sua

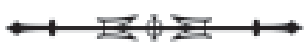


área de distribuição é relativamente ampla e encontra-se no Brasil, Colômbia, Peru e Venezuela (AYTUARD; CUELLO; FERNÁNDEZ, 1990). No Brasil ocorre nos estados do Acre, Amazonas, Pará e Roraima. No Acre distribui-se nos municípios de Cruzeiro do Sul, Mâncio Lima, Rio Branco e Sena Madureira (Figura 3). Obedece a uma distribuição particular, sempre concentrada em locais de solos arenosos e bem drenados.

\section{Status de conservação}

Esta espécie foi incluída na categoria CR (perigo crítico), por ter uma distribuição particular. As plantas das populações ainda existentes estão sendo eliminadas devido ao seu potencial uso medicinal, madeireiro e para a ampliação da fronteira agropecuária (ALARCÓN; MENA, 1994). Sua presença em unidades de conservação é desconhecida e poucas são as coletas reportadas para esta espécie.

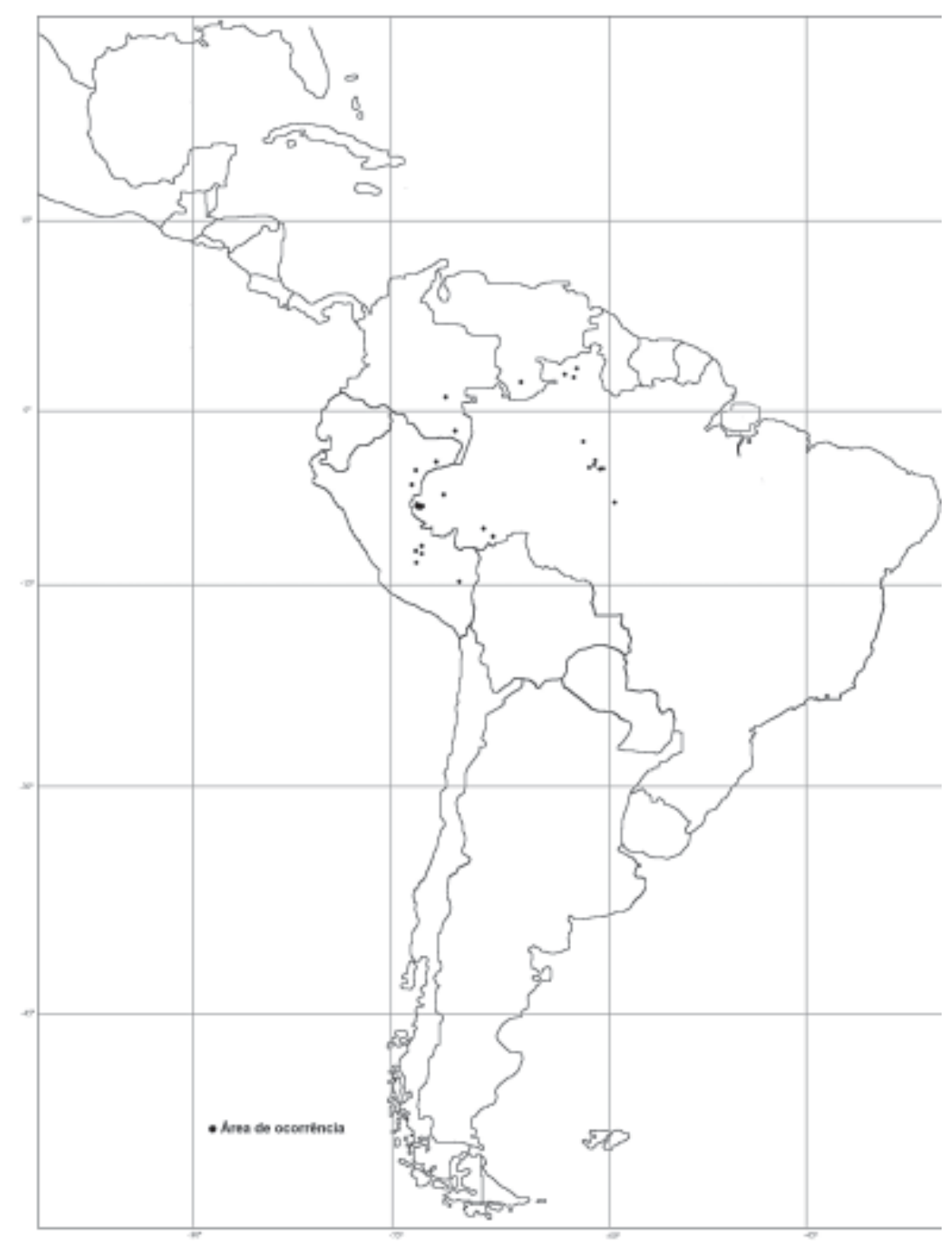

Figura 3. Distribuição geográfica de Cinchona amazonica Standl. na América Latina.

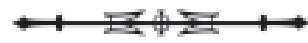




\section{CONCLUSÕES}

A descrição dendrológica de Cinchona amazonica Standl. foi consolidada, no presente trabalho, através da análise das suas características reprodutivas e vegetativas, fundamentadas no material botânico coletado em sua área de ocorrência natural, em herbários de 3 países e na literatura especializada.

Optou-se pela manutenção da denominação original do gênero Cinchona, ao invés de Cinchonopsis, proposto por Andersson, por considerar a necessidade da inclusão de maior número de exsicatas procedentes e representativas de áreas mais amplas de ocorrência da espécie e que incorporem a sua variabilidade.

Cinchona amazonica mostrou uma ampla distribuição geográfica no Brasil e na América sendo, no entanto, incluída na categoria de perigo crítico pelo seu emprego como planta medicinal e por sua área de ocorrência estar sendo submetida a intensa pressão para uso agrícola.

Evidenciou-se, também, a necessidade da intensificação dos estudos sobre a espécie, com a ampliação da área de coleta de materiais botânicos e aplicação de métodos complementares de identificação para a definição do seu taxon.

\section{AGRADECIMENTOS}

Aos Herbários do Brasil (ESA, HPZ, HRCB, IAN, INPA, MG, R, RB, SP, SPF, UEC e UNBA), da Bolívia (LPB) e do Peru (AMAZ, MOL e USM), pelo apoio na revisão das exsicatas do gênero estudado.

\section{REFERÊNCIAS}

ALARCÓN, R.; MENA, P. 1994. Etnobotánica, valoración económica y comercialización de recursos florísticos silvestres en el alto NapoEcuador. Ecociencia, v. 1, p. 161-162.

ANDERSSON, L. A. 1995. Tribes and genera of the Cinchoneae complex (Rubiaceae). In: INTERNATIONAL CONFERENCE ON THE RUBIACEAE, 1., 1993, St. Louis Missouri. Annals of the Missouri Botanical Garden. St. Louis Missouri: [s.n.]. v. 82. n. 3. p. 409-427.
ANDERSSON, L. A. 1998. Revision of the genus Cinchona (RubiaceaeCinchoneae). Memories the New York Botanical Garden, v. 80, p. 1-75.

ANDERSSON, L. A.; PERSSON, C. 1991. Circunscription of the tribe Cinchoneae Rubiaceae-A cladistic approach. Plant Systematics and Evolution, v. 178, p. 65-94.

AYNARD, G. A.; CUELLO, N.L.; FERNÁNDEZ A. 1990. Observaciones sobre hallazgo de Cinchona amazonica Standl. (Rubiaceae) en la Guyana Venezolana. Biollania, v. 7, p. 125-130. BARCELÓ, J.; NICOLÁS, G.; SABATER, B.; SÁNCHEZ, R. 2001. Fisiologia Vegetal. Madrid, España: Pirámide. v. 1.

BARQUERO. 1986. El cultivo del árbol de la Quina. Costa Rica: Colegio de Ingeniero grónomos. 13 p.

BREMER, B.; ANDREASEN, K.; OLSSON, D. 1995. Subfamilial and tribal relationships in the Rubiaceae based on RBCL sequence data. In: INTERNATIONAL CONFERENCE ON THE RUBIACEAE, 1., Annals of the Missouri Botanical Garden, v. 82, n. 3 , p. 383-397.

BREMER, K.; BREMER, B.; THULIN, E. 1998. A ordinal classification for the families of flowering plants. Annals of The Missouri Botanical Garden, v. 85, p. 531-553.

BRUNETON, J. 2001. Farmacognosia, fitoquímica, plantas medicinales. 2. ed. Zaragoza, España: Acribia. 1100 p.

BURGER, W. 1993. Rubiaceae: flora Costariquensis. Fieldiana, v. 33, p. 102-103

CARVALHO, A.; KRUG, C. A. 1994. A quineira (Cinchona sp.): origem, classificação, exploração econômica no mundo e tentativas de sua aclimatação no Brasil. Campinas: IAC. 141 p.

CDC. 1991. Centro de datos para la conservación. Plan Director del Sistema Nacional de Unidades de Conservación (Sinuc), una aproximación desde la diversidad biológica. Lima, Perú: Universidad Nacional Agraria La Molina. 153 p.

CRONQUIST, A. 1988. The evolution and classification of flowering plants. 2. ed. New York: The New York Botanical Garden Press. 555 p.

DECANDOLLE. 1797. A description of the Genus Cinchona. London: British Museum. .4144 p.

DELPRETE, P. G.; CORTÉS, R. 2002. Rubiaceae del Nuevo Mundo. In: CONGRESO LATINOAMERICANO DE BOTÁNICA, 8., Colombia, Cartagena de Indias: Resúmenes del VIII-CLB ... Santafé de Bogotá, Colombia: ALB, ACB, ACH, ICN. p. 417.

DÉTIENNE, P.; JACQUET, P. 1983. Atlas d'identification des bois de l'amazonie et des régions voisines. Montpellier, Francia: Centre Technique Forestier Tropical. 640 p.

GENTRY A. 1993. A field guide to the families and genera of woody plants of northwest Southamerica. Washington, DC.: Conservation Internacional. $411 \mathrm{p}$.

GUPTA, M. F. 1995. Plantas medicinais iberoamericanas. Santafé de Bogotá, Colombia: Editorial Presencia. p. 486-487.

HUTCHINSON, J. 1967. The genera of flowering plantsAngiospermae: dicotyledones. Oxford, United Kingdow: Oxford University Press. v. 2.

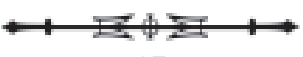


IMAC. 1991. Instituto de meio ambiente do acre. Atlas geográfico ambiental do Acre. Rio Branco: Secretaria de Meio Ambiente do Acre. v. 1.

IUCN. 1993. WWF International Union For Conservation Of NatureWorld Wide Fund For Nature. Guidelines for the Conservation of Medicinal Plants. Gland, Switzerland: IUNC. v. 1.

IUCN. 1998. International Union For Conservation Of Nature. The IUCN Red List of Threatened Plants. Gland, Switzerland: IUCN. v. 1

IUCN. 2002. International Union For Conservation Of Nature. The IUCN Red List of Threatened Plants. Version 3.1. Gland. Switzerland: IUCN. v. 1. (Species Survival Commission).

MENEZES, M. A. 1994. As reservas extrativistas como alternativa do desmatamento na Amazônia. In: ARNT R. O Destino da floresta-reservas extrativistas e desenvolvimento sustentável na Amazônia. Rio de Janeiro: Relume Dumará. p. 49-72.

OCAMPO-SANCHEZ, R. A. 2000. Agrotecnología para el cultivo de quina o cascarilla. In: MARTÍNEZ J. V.; BERNAL, H. J.; CÁCERES, A. Fundamentos de agrotecnología para el cultivo de plantas medicinais Iberoamericanas. Santafé de Bogotá, Colombia: Convenio Andrés Bello/Ciencia y Tecnología para el Desarrollo. p. 147-156.

PINO, G. D. 2002. Caracterização dendrologica de las Rubiaceae de los bosques de Chanchamayo, JunínPerú. 210 f. Tese (Doutorado) - Universidad Nacional Agraria La Molina, Lima.

PRINZ, A. 1990. Discovery of the cardiac effectiveness of Cinchona bark and its alkaloids. Wien Klin Wochenschr, n. 102, v. 24, p. $721-723$

RADFORD, A. E. et al. 1974. Vascular plant systematics. New York: Harper and Row. 891 p.
REA, R. L. 1995. Cinchona y la tribu Cinchoneae (Rubiaceae) en Bolivia, actualización sistemática, fitoquímica y actividad antimalárica. 183 f. Tese (Doutorado) - Universidade Mayor de San Andrés de La Paz-Bolívia, La Paz, Bolívia.

ROBBRECHT, E. 1988. Tropical Woody Rubiaceae. Opera Botanica Belga, v. 1, p.1-271.

SCHULTES, R. E. 1979. Indícios da riqueza etnofarmacológica do noroeste da Amazônia. Acta Amazonica, v. 91, n. 1, p. 209-215.

STELL, R. 1982. Flores para el Rey. Barcelona,España: Ed. Serbal. $347 \mathrm{p}$.

STEYERMARK, J. A. 1974. Rubiaceae. In: LASER, T. Flora de Venezuela. Caracas, Venezuela: Instituto Botánico, Dirección de Recursos Naturales Renovables, Ministerio de Agricultura y Cria, Caracas. v. 91. n. 1. p. 1-2070.

VAN-HARTEN, A. M. 1987. Quina Cinchona spp. In: GEOTECNIA de Cultivos Tropicales Perennes. [S.l.]: AGT Editor. p. 438-455.

VERPOORTE, R.; SCHRIPSEMA, J.; VAN-DER-LEER, T. 1988.

Cinchona Alkaloid. Brossi. The Alkaloids, Chemistry and Pharmacology, v. 34, p. 331-398.

VERVEEN, G. 1984. La Malaria. Hexágono-Roche, v. 2, n. 3, p. 11-16. WARHURST, D. C. 1987. Cinchona alkaloids and malaria. Acta Leidensia, v. 55, p. 55-64.

ZEVALLOS, P. P. 1989. Taxonomía, distribuición geográfica y status del gênero Cinchona en el Peru. Lima, Peru: Centro de Datos Para la Conservación, Universidade Nacional Agraria la Molina. 87 p.

ZEVALLOS, P. P. 2004. Dendrologia, anatomia do lenho e "status" de conservação das espécies lenhosas dos gêneros Cinchona, Croton e Uncaria no Estado do AcreBrasil. 181 f. Tese (Doutorado) - Escola Superior de Agricultura Luiz de Queiroz-Universidade de São Paulo, São Paulo. 